\title{
The Universal Parabola
}

\section{The Universal Parabola \\ ABSTRACT}

We develop classical properties, as well as some novel facts, for the parabola using the more general framework of rational trigonometry. This extends the study of this conic to general fields.

Key words: parabola, rational trigonometry, conic

MSC2010: 51N20, 14H50

\section{Introduction}

Next to the circle, the parabola is perhaps the most accessible conic section. It was studied by Menaechmus, who used it to duplicate the volume of a cube. Apollonius gave it its name, and deduced many important properties. Archimedes studied areas of parabolic arcs, Euclid mentions the parabola, and Pappus investigated the focus and directrix. Galileo showed that projectiles follow parabolic arcs. The reflective property was studied by Gregory and Newton. The parabola appears in car headlights, solar ovens, telescopes, astronomical radio dishes, the orbits of comets, architecture and whenever one variable is proportional to the square of another.

Classical geometry considers the parabola to be an element of Euclidean geometry over the field of decimal or 'real' numbers. Treatises which establish some of these properties include [3], [4], [6], [7], [8], [9], [10], [11], [12], [13] and [14]. From our point of view, this traditional aspect is but a shadow of the true parabola, which is an object that properly lives in universal geometry, a form of Euclidean metrical geometry that is valid over a general field. There are parabolas defined over finite fields, over the complex numbers, and over the p-adic numbers. With universal geometry we may investigate properties of parabolas that are shared in these different contexts, in other words that hold in complete generality.

\section{Univerzalna parabola \\ SAŽETAK}

Dokazujemo neka klasična svojstva kao i neke nove činjenice o paraboli koristeći okvir racionalne trigonometrije. Proširujemo proučavanje konika na opća polja.

Ključne riječi: parabola, racionalna trigonometrija, konika

This may well strike the reader as curious. One of the most familiar properties of a parabola is the reflective propertyany light beam coming in parallel to the axis and reflected off the parabola so as to make equal angles with the tangent line passes through the focus. How is one to even state such a fact over say the finite field $\mathbb{F}_{11}$ where angles make no sense?

The answer is to free oneself from the straightjacket of traditional geometric thought. Distance and angle are not really the mathematically fundamental concepts that we like to believe. Euclid carefully avoided mentioning these metrical notions because of their attendant irrationalities. Make the shift to quadrance and spread, and you have an entirely new and simplified way of thinking about metrical geometry, which allows you to study parabolas and other conic sections in the universal setting, as well as much else besides, as shown in the recent book [16]. This can then be extended also to hyperbolic geometry, as in [1] and [2].

This paper derives numerous properties of the universal parabola. Some of the theorems are extensions of familiar and classical results, suitably restated in the new language to hold in an arbitrary field. Others are new even in the familiar setting. The diagrams mostly illustrate the situation in the familiar domain of the rational numbers, or numerically the decimal number field. 


\section{Universal geometry}

Universal geometry, introduced in [16], is a form of Euclidean geometry that holds over any field, characteristic two (and sometimes three) excluded. Distance and angle are replaced by algebraic analogs; the separation of points is measured using quadrance, and the separation of lines is measured using spread. The following definitions and results are taken from [16].

Fix a field $\mathbb{F}$ whose elements are called numbers. We will throughout assume that the characteristic of this field is not two. That means that the number 2 is always invertible in $\mathbb{F}$. A point is an ordered pair of numbers, denoted $A \equiv[x, y]$. A side $\overline{A_{1} A_{2}}$ is a set consisting of two points $A_{1}$ and $A_{2}$. The midpoint of the side $\overline{A_{1} A_{2}}$, where $A_{1}=\left[x_{1}, y_{1}\right]$ and $A_{2}=\left[x_{2}, y_{2}\right]$, is the point

$M=\left[\frac{x_{1}+x_{2}}{2}, \frac{y_{1}+y_{2}}{2}\right]$.

A line is a proportion $(a: b: c)$ of numbers with at least one of $a$ and $b$ non-zero. The point $A \equiv[x, y]$ lies on the line $l \equiv(a: b: c)$ precisely when $a x+b y+c=0$, which is called the equation of the line. Equivalently we say $l$ passes through $A$.

A line $(a: b: c)$ is a null line precisely when $a^{2}+b^{2}=0$. Null lines do not occur over the rational or decimal number fields, but they occur whenever -1 is a square. In fact if $i^{2}=-1$ then any null line has the form $(1: \pm i: c)$ for some $c$.

Theorem 1 (Collinear points) The points $\left[x_{1}, y_{1}\right],\left[x_{2}, y_{2}\right]$ and $\left[x_{3}, y_{3}\right]$ are collinear (meaning they lie on the same line) precisely when

$x_{1} y_{2}-x_{1} y_{3}+x_{2} y_{3}-x_{3} y_{2}+x_{3} y_{1}-x_{2} y_{1}=0$.

Theorem 2 (Concurrent lines) If the lines $\left(a_{1}: b_{1}: c_{1}\right)$, $\left(a_{2}: b_{2}: c_{2}\right)$ and $\left(a_{3}: b_{3}: c_{3}\right)$ are concurrent (meaning they pass through the same point) then

$a_{1} b_{2} c_{3}-a_{1} b_{3} c_{2}+a_{2} b_{3} c_{1}-a_{3} b_{2} c_{1}+a_{3} b_{1} c_{2}-a_{2} b_{1} c_{3}=0$.

Definition 1 The lines $l_{1}=\left(a_{1}: b_{1}: c_{1}\right)$ and $l_{2} \equiv$ $\left(a_{2}: b_{2}: c_{2}\right)$ are parallel precisely when

$a_{1} b_{2}-a_{2} b_{1}=0$.

Definition 2 The lines $l_{1}=\left(a_{1}: b_{1}: c_{1}\right)$ and $l_{2} \equiv$ $\left(a_{2}: b_{2}: c_{2}\right)$ are perpendicular precisely when

$a_{1} a_{2}+b_{1} b_{2}=0$.

Definition 3 The quadrance $Q\left(A_{1}, A_{2}\right)$ between the points $A_{1} \equiv\left[x_{1}, y_{1}\right]$ and $A_{2} \equiv\left[x_{2}, y_{2}\right]$ is the number

$Q\left(A_{1}, A_{2}\right) \equiv\left(x_{2}-x_{1}\right)^{2}+\left(y_{2}-y_{1}\right)^{2}$.
Definition 4 The spread $s\left(l_{1}, l_{2}\right)$ between the non-null lines $l_{1}=\left(a_{1}: b_{1}: c_{1}\right)$ and $l_{2} \equiv\left(a_{2}: b_{2}: c_{2}\right)$ is the number

$s\left(l_{1}, l_{2}\right) \equiv \frac{\left(a_{1} b_{2}-a_{2} b_{1}\right)^{2}}{\left(a_{1}^{2}+b_{1}^{2}\right)\left(a_{2}^{2}+b_{2}^{2}\right)}$.

Two lines $l_{1}$ and $l_{2}$ are perpendicular precisely when $s\left(l_{1}, l_{2}\right)=1$. If $\overline{A_{1} A_{2} A_{3}}$ is a right triangle with $A_{1} A_{3}$ perpendicular to $A_{2} A_{3}$, then the Spread ratio theorem asserts that

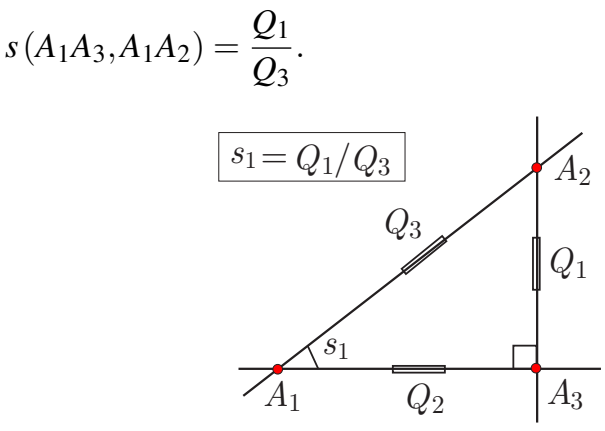

Figure 1: Spread as ratio

For any point $A \equiv[x, y]$ and any line $l \equiv(a: b: c)$ there is a unique line $n$, called the altitude from $A$ to $l$, which passes through $A$ and is perpendicular to $l$, namely

$n=(-b: a: b x-a y)$.

If $l$ is non-null, then this altitude intersects $l$ at the point

$N \equiv\left[\frac{b^{2} x-a b y-a c}{a^{2}+b^{2}}, \frac{-a b x+a^{2} y-b c}{a^{2}+b^{2}}\right]$

called the foot of the altitude. The quadrance $Q(A, l)$ between the point $A$ and the line $l$ is then defined to be $Q(A, N)$. This turns out to be

$Q(A, l)=\frac{(a x+b y+c)^{2}}{a^{2}+b^{2}}$.

\section{Isometries and similarities}

Definition 5 An isometry $\sigma$ is a function that inputs and outputs points, such that for any points $A_{1}$ and $A_{2}$

$Q\left(A_{1}, A_{2}\right)=Q\left(\sigma\left(A_{1}\right), \sigma\left(A_{2}\right)\right)$.

Definition 6 A similarity $\tau$ is a function that inputs and outputs points, such that for any points $A_{1}, A_{2}$ and $A_{3}$,

$Q\left(A_{1}, A_{2}\right) Q\left(\tau\left(A_{2}\right), \tau\left(A_{3}\right)\right)=Q\left(A_{2}, A_{3}\right) Q\left(\tau\left(A_{1}\right), \tau\left(A_{2}\right)\right)$.

Every isometry is a similarity, but scalings are similarities which are not generally isometries. A similarity preserves the ratio of quadrances, so it preserves the spread between lines. 
Theorem 3 For any numbers $a$ and $b$, the function

$\tau([x, y])=[a x+b y, b x-a y]$

is a similarity.

Proof. Suppose that $A_{1}=\left[x_{1}, y_{1}\right], A_{2}=\left[x_{2}, y_{2}\right]$. Then

$$
\begin{aligned}
& Q\left(\tau\left(A_{1}\right), \tau\left(A_{2}\right)\right) \\
& =Q\left(\left[a x_{1}+b y_{1}, b x_{1}-a y_{1}\right],\left[a x_{2}+b y_{2}, b x_{2}-a y_{2}\right]\right) \\
& =\left(a x_{2}+b y_{2}-a x_{1}-b y_{1}\right)^{2}+\left(b x_{2}-a y_{2}-b x_{1}+a y_{1}\right)^{2} \\
& =\left(a^{2}+b^{2}\right)\left(x_{1}^{2}-2 x_{1} x_{2}+x_{2}^{2}+y_{1}^{2}-2 y_{1} y_{2}+y_{2}^{2}\right) \\
& =\left(a^{2}+b^{2}\right) Q\left(A_{1}, A_{2}\right)
\end{aligned}
$$

from which the result follows.

The transformation $\tau$ above is affine, implying that lines are sent to lines. The point $[x, y]$ lies on the line $a x+b y+$ $c=0$ precisely when $\tau([x, y])$ lies on the line $x+c=0$. The inverse transformation to $\tau$ is defined only if $a^{2}+b^{2} \neq 0$ and is

$$
\tau^{-1}([x, y])=\left[\frac{a x+b y}{a^{2}+b^{2}}, \frac{b x-a y}{a^{2}+b^{2}}\right] .
$$

\section{Parabolas}

A conic is given by a polynomial equation in $x$ and $y$ of degree exactly two. A circle is a conic whose equation in $X \equiv[x, y]$ has the form $Q(X, C)=R$ for some point $C$ and some number $R$. Some basic facts about circles will be taken from [16], such as the fact that if $A$ and $B$ lie on the circle, then the spread $s(A X, B X)$ is constant for all $X$ lying on the circle, and is equal to 1 precisely when $A B$ is a diameter of the circle (i.e. passes through the center $C$ ).

Definition 7 A parabola $p$ is a conic whose equation in $X \equiv[x, y]$ has the form

$$
Q(X, F)=Q(X, l)
$$

for some fixed point $F$ and some fixed non-null line $l$ not passing through $F$.

A point $A=[x, y]$ lies on $p$ precisely when $x$ and $y$ satisfy the equation of $p$. It is a theorem in [16] that the point $F$ and the line $l$ are determined by $p$. The point $F$ is the focus and the line $l$ the directrix of $p$. The axis of the parabola $p$ is the altitude from $F$ to $l$. The vertex of the parabola is the midpoint of the side $\overline{F P}$ where $P$ is the foot of the altitude from $F$ to the directrix $l$. It lies on the axis and also on the parabola $p$.

Example 1 If $\lambda \neq 0$ then the parabola $p$ with focus $F \equiv$ $[\lambda, 0]$ and directrix $x+\lambda=0$ has equation

$(x-\lambda)^{2}+y^{2}=(x+\lambda)^{2} \quad$ or $\quad y^{2}=4 \lambda x$.
Every point lying on this parabola has the form $\left[\lambda a^{2}, 2 \lambda a\right]$ for some number $a$, and every such point lies on the parabola.

To prove theorems about parabolas, it is very convenient to work with this simple equation. The following useful result shows how this can be done.

Theorem 4 Any parabola can be transformed by a similarity to one of the form $y^{2}=4 \lambda x$ for some number $\lambda$.

Proof. If the directrix of the parabola $p$ is $a x+b y+c=0$ then the similarity

$$
\tau([x, y])=[a x+b y, b x-a y]
$$

takes this line to $x+c=0$. This transformation is invertible since the directrix of a parabola is a non-null line, so that $a^{2}+b^{2} \neq 0$. A translation (in the $y$ direction) now moves the focus to the form $[\beta, 0]$ and leaves the directrix unchanged. Then the translation (in the $x$ direction)

$$
[x, y] \rightarrow[x-(\beta-c) / 2, y]
$$

takes the focus to $[(\beta+c) / 2,0]$ and the directrix to $x+$ $(\beta+c) / 2=0$ which has the required form, with $\lambda=$ $(\beta+c) / 2$.

Although the transformation used here is a similarity, not in general an isometry, it is sufficient to prove all the theorems in this paper, which are ultimately not about individual quadrances, but always only about the proportions between quadrances.

The parabola $p$ with equation $y^{2}=4 \lambda x$ has focus $F=[\lambda, 0]$, directrix $(1: 0: \lambda)$, axis the line $(0: 1: 0)$ and vertex the point $[0,0]$. If $A$ and $B$ are two distinct points lying on $p$, then the line $A B$ is a chord of the parabola, while the side $\overline{A B}$ is a side of the parabola. A chord passing through the focus $F$ is a focal chord. The chord of a parabola through its focus which is perpendicular to its axis is called the latus rectum. A null point of $p$ is a point $N$ lying on $p$ such that $Q(N, F)=0$.

Tangent lines to conics are best defined algebraically, since limiting procedures are not available for general fields. Given a conic which passes through the origin with equation such as $x^{2}-3 x y+5 y^{2}+3 x-2 y=0$, the tangent line at the origin is defined to be just the linear part of this expression, that is the line $3 x-2 y=0$. To define the tangent at a general point of a general conic, first translate so that the point is at the origin, then take the linear part, and translate back. This is explained in more detail in [16], which also contains the following result, generalizing the most well-known property of a parabola to the universal case. 
Theorem 5 Suppose $k$ is the tangent line to a parabola $p$ at a point $A$ lying on it. Then $s(k, m)=s(k, n)$ where $m=A F$ with $F$ the focus of $p$, and $n$ is the altitude from $A$ to the directrix of $p$.

The general point on $y^{2}=4 \lambda x$ is $\left[x_{1}, y_{1}\right]=\left[\lambda t^{2}, 2 \lambda t\right]$ in terms of a parameter $t$. The tangent to $p$ at this point is the line $\left(2 \lambda:-y_{1}: 2 \lambda x_{1}\right)=\left(1:-t: \lambda t^{2}\right)$. A null tangent to a parabola is a null line that is tangent to the parabola, clearly this occurs precisely when $t^{2}=-1$. For two different values of $t$, say $t=a$ and $t=b$ we get two points $A \equiv\left[\lambda a^{2}, 2 \lambda a\right]$ and $B \equiv\left[\lambda b^{2}, 2 \lambda b\right]$, which determine the chord $(2:-(a+b): 2 \lambda a b)$. The tangents to these points intersect at the point

$X=\left[x_{0}, y_{0}\right]=[\lambda a b, \lambda(a+b)]$

which is the external point of the chord $A B$. The side $\overline{A B}$ is the side determined by $X$, and the chord $A B$ is the chord of contact of $X$; it has the form $\left(2 \lambda:-y_{0}: 2 \lambda x_{0}\right)$.

Summary of formulae Let $p$ be the parabola with equation $y^{2}=4 \lambda x$. Let $\left[x_{1}, y_{1}\right]$ denote a general point on the parabola, and $A \equiv\left[\lambda a^{2}, 2 \lambda a\right]$ and $B \equiv\left[\lambda b^{2}, 2 \lambda b\right]$ two specific points on the parabola. Let $\left[x_{0}, y_{0}\right]$ be the external point of the chord $A B$. Let $\left[\lambda t^{2}, 2 \lambda t\right]$ be the parametric form of $p$.

The tangent to $p$ at $\left[x_{1}, y_{1}\right]$ in Cartesian form

$\left(2 \lambda:-y_{1}: 2 \lambda x_{1}\right)$

The tangent to $p$ at $A=\left[\lambda a^{2}, 2 \lambda a\right]$ in parametric form

$\left(1:-t: \lambda t^{2}\right)$

The chord $A B$ in Cartesian form

$\left(2 \lambda:-y_{0}: 2 \lambda x_{0}\right)$

\section{The chord $A B$ in parametric form}

$(2:-(a+b): 2 \lambda a b)$

The external point of the chord $A B$ in parametric form

$[\lambda a b, \lambda(a+b)]$

\section{Tangents and external points}

Theorem 6 No two tangents to a parabola are parallel.
Proof. Let the equation of the parabola be

$y^{2}=4 \lambda x$

for some $\lambda \neq 0$. Then the tangents to the parabola at two distinct points $\left[x_{1}, y_{1}\right],\left[x_{2}, y_{2}\right]$ are

$$
\left(2 \lambda:-y_{1}: 2 \lambda x_{1}\right) \text { and }\left(2 \lambda:-y_{2}: 2 \lambda x_{2}\right)
$$

respectively. But since $y_{1} \neq y_{2}$, these lines are not parallel.

Theorem 7 No three tangents to a parabola are concurrent.

Proof. Since affine transformations preserve concurrence of lines, it suffices to prove this for the parabola

$y^{2}=4 \lambda x$.

Let

$$
A \equiv\left[\lambda a^{2}, 2 \lambda a\right] \quad B \equiv\left[\lambda b^{2}, 2 \lambda b\right] \quad \text { and } \quad C \equiv\left[\lambda c^{2}, 2 \lambda c\right] .
$$

The tangents to the parabola at these points are

$$
\left(1:-a: \lambda a^{2}\right) \quad\left(1:-b: \lambda b^{2}\right) \text { and }\left(1:-c: \lambda c^{2}\right)
$$

respectively. Suppose the three tangents are concurrent, then by the Concurrent lines theorem

$$
\left|\begin{array}{lll}
1 & -a & \lambda a^{2} \\
1 & -b & \lambda b^{2} \\
1 & -c & \lambda c^{2}
\end{array}\right|=0
$$

Expand the determinant to get

$\lambda(a-b)(c-a)(c-b)=0$

Since $A, B$ and $C$ are all distinct points, $a, b$ and $c$ are also distinct. This implies $\lambda=0$, which is impossible.

Theorem 8 There is a unique pair of tangents to a parabola through an external point.

Proof. By Theorem 7 no three tangents are concurrent, hence there is no external point with two distinct pairs of tangents to the parabola.

\section{Chords of a parabola}

Theorem 9 Suppose $A$ and $B$ are two points on $a$ parabola. Then the following are equivalent.

\section{AB is a focal chord}

2. The tangents at $A$ and $B$ are perpendicular

3. The tangents at $A$ and $B$ intersect on the directrix. 


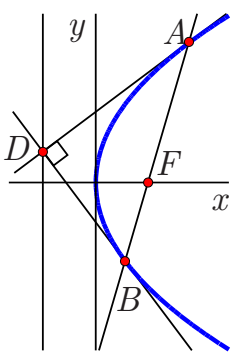

Figure 2.

Proof. $1 \Rightarrow 2$ ) Let $A \equiv\left[\lambda a^{2}, 2 \lambda a\right]$ and $B \equiv\left[\lambda b^{2}, 2 \lambda b\right]$ and $\left[x_{0}, y_{0}\right]$ be the external point of the chord $A B$. By (2) the tangents to $p$ at $A$ and $B$ are

$$
\left(1:-a: \lambda a^{2}\right) \text { and }\left(1:-b: \lambda b^{2}\right)
$$

respectively. Since the line $A B$ passes through the focus $[\lambda, 0]$, from (4) we deduce that

$$
a b=-1 .
$$

Hence the two tangents are perpendicular.

$2 \Rightarrow 3$ ) If the tangents are perpendicular, then from (2) $a b=-1$. By comparing (4) with (3), $x_{0}=-\lambda$. Therefore the external point of the chord $A B$ lies on the directrix. Hence the two tangents intersect on the directrix.

$3 \Rightarrow 1$ ) Suppose two tangents to the parabola intersect on the directrix i.e. $x_{0}=-\lambda$. Then from (3) the chord $A B$ is

$$
\left(2 \lambda:-y_{0}:-2 \lambda^{2}\right)
$$

which passes through the point $[\lambda, 0]$. Hence $A B$ is a focal chord.

Theorem 10 Any circle whose diameter is a focal chord of a parabola touches the directrix.

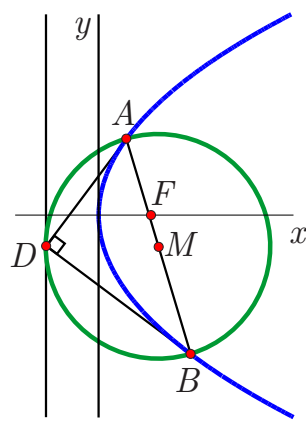

Figure 3.

Proof. Since the tangents to $p$ at $A=\left[\lambda a^{2}, 2 \lambda a\right]$ and $B=\left[\lambda b^{2}, 2 \lambda b\right]$ intersect at

$D \equiv[\lambda a b, \lambda(a+b)]$ we may compute that

$A D=\left(1:-a: a^{2} \lambda\right) \quad$ and $\quad B D=\left(1:-b: b^{2} \lambda\right)$.

If $A B$ is a focal chord then from the proof of the previous proposition, $a b=-1$,so that $A D$ and $B D$ are perpendicular. Then $D$ lies on the circle with $A B$ as its diameter. In particular, the midpoint

$M \equiv\left[\frac{\lambda\left(a^{2}+b^{2}\right)}{2}, \lambda(a+b)\right]$

of the side $\overline{A B}$ is the center of the circle. The equation of the line $M D$ is

$(0: 1:-\lambda(a+b))$

which is perpendicular to the directrix. Therefore the directrix is tangent to the circle.

Theorem 11 Let $A$ be a point on a parabola with focus $F$. Let $D$ be the foot of the altitude from $A$ to the directrix, and $T$ be the point where the tangent at A meets the axis of the parabola. Then the side $\overline{F D}$ is perpendicular to the side $\overline{A T}$ and they share a common midpoint.

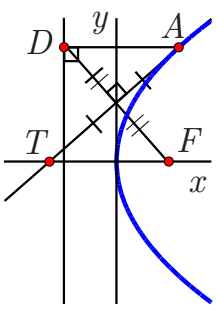

Figure 4.

Proof. Let $A \equiv\left[\lambda a^{2}, 2 \lambda a\right]$. By (2) the tangent at $A$ is

$\left(1:-a: \lambda a^{2}\right)$

which meets the axis at

$T=\left[-\lambda a^{2}, 0\right]$.

By the foot of an altitude formula

$D=[-\lambda, 2 \lambda a]$

So the line $A T$ and the line

$D F=(a: 1:-\lambda a)$

are perpendicular. Moreover the midpoints of the side $\overline{A T}$ and $\overline{D F}$ are both

$[0, \lambda a]$.

Theorem 12 Let $A B$ be a chord of a parabola which is parallel to the directrix. Let $E$ be a third point on the parabola. Suppose the lines EA and EB intersect the axis of the parabola at $C$ and $D$ respectively. Then the midpoint of the side $\overline{C D}$ is the vertex of the parabola. 


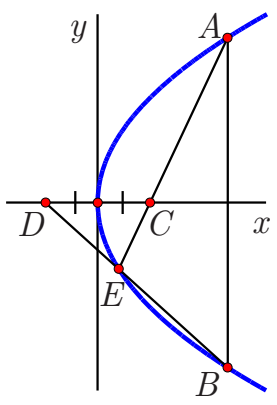

Figure 5.

Proof. Let

$A \equiv\left[\lambda a^{2}, 2 \lambda a\right] \quad B \equiv\left[\lambda b^{2}, 2 \lambda b\right] \quad$ and $\quad E \equiv\left[\lambda e^{2}, 2 \lambda e\right]$

Since the line $A B$ is parallel to the directrix by (4)

$a+b=0$.

From (4) the line $E A$ intersects the axis of parabola at

$C \equiv[-\lambda e a, 0]$

Similarly the line $E B$ intersects the axis of the parabola at

$D \equiv[-\lambda e b, 0]$

Therefore the midpoint of $\overline{C D}$ is

$\left[\frac{-\lambda e(a+b)}{2}, 0\right]=[0,0]$

which is the vertex of the parabola.

Theorem 13 Let $\overline{A B}$ be the side of an external point $X$ to a parabola. Let $M$ be the midpoint of the side $\overline{A B}$. Then

1. MX is parallel to the axis of the parabola.

2. The midpoint $T$ of the side $\overline{M X}$ lies on the parabola.

3. The tangent at $T$ is parallel to the chord $A B$.

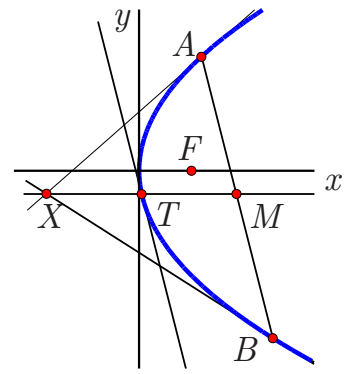

Figure 6.
Proof. 1.) Let $A \equiv\left[\lambda a^{2}, 2 \lambda a\right]$ and $B \equiv\left[\lambda b^{2}, 2 \lambda b\right]$. Then the midpoint of the side $\overline{A B}$ is

$M=\left[\frac{\lambda\left(a^{2}+b^{2}\right)}{2}, \lambda(a+b)\right]$.

By (5) the external point of $\overline{A B}$ is

$X=[\lambda a b, \lambda(a+b)]$

which has the same $y$ component as $M$. Hence $M X$ is parallel to the axis $(0: 1: 0)$ of the parabola.

2.) The midpoint $T$ of the side $\overline{M X}$ is

$\left[\frac{\lambda(a+b)^{2}}{4}, \lambda(a+b)\right]$

which lies on the parabola, with parameter $t=(a+b) / 2$.

3.) By (2) the tangent at $T$ is

$\left(4:-2(a+b): \lambda(a+b)^{2}\right)$.

By (4) the line containing the chord is

$(2:-(a+b): 2 \lambda a b)$

which is parallel to the tangent at $T$.

Theorem 14 Let $A B$ be the chord from an external point $X$ to a parabola with focus $F$. Suppose the line $A B$ meets the axis at $I$, and that the line through $X$ parallel to the axis meets the parabola at $N$. Then

1. The midpoint of the side $\overline{X I}$ lies on the tangent at the vertex of parabola.

2. The tangent at $N$ is parallel to the chord $A B$.

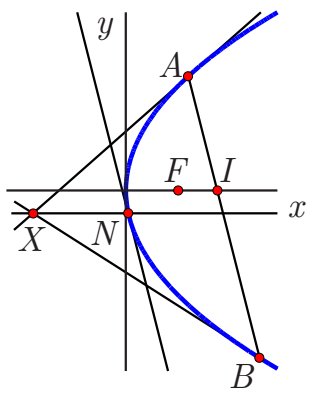

Figure 7.

Proof. Let $A \equiv\left[\lambda a^{2}, 2 \lambda a\right]$ and $B \equiv\left[\lambda b^{2}, 2 \lambda b\right]$ as usual. 1.) $\mathrm{By}(4)$

$I=[-\lambda a b, 0]$

and by (5)

$X=[\lambda a b, \lambda(a+b)]$

Therefore the midpoint of the side $\overline{X I}$ is

$\left[0, \frac{\lambda(a+b)}{2}\right]$ 
which lies on $(1: 0: 0)$, the tangent at the vertex to the parabola.

2.) The line through $X$ parallel to the axis of the parabola intersects the parabola at

$N \equiv\left[\frac{\lambda(a+b)^{2}}{4}, \lambda(a+b)\right]$.

By (1) the tangent at $N$ is

$\left(4:-2(a+b): \lambda(a+b)^{2}\right)$

which is parallel to line $A B$ from (4).

Theorem 15 For any point $C$ there is a line $l=l(C)$ with the property that if a chord $A B$ of the parabola $p$ passes through $C$, then the external point of the chord lies on $l$.

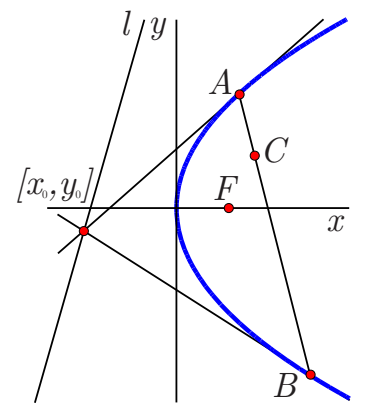

Figure 8 .

Proof. Let the external point of the chord be $\left[x_{0}, y_{0}\right]$ and $C \equiv[h, k]$. By assumption the chord passes through $[h, k]$, so by (3)

$2 \lambda h-y_{0} k+2 \lambda x_{0}=0$.

Hence $\left[x_{0}, y_{0}\right]$ lies on the line

$l \equiv(2 \lambda:-k: 2 \lambda h)$.

$C$ is called the pole of $l$, while $l$ is called the polar of $C$.

Theorem 16 Let $A_{1} \equiv\left[x_{1}, y_{1}\right]$ and $A_{2} \equiv\left[x_{2}, y_{2}\right]$ be two external points to a parabola. If the line containing the chord of contact from $A_{1}$ passes through $A_{2}$, then the line containing the chord of contact from $A_{2}$ passes through $A_{1}$.

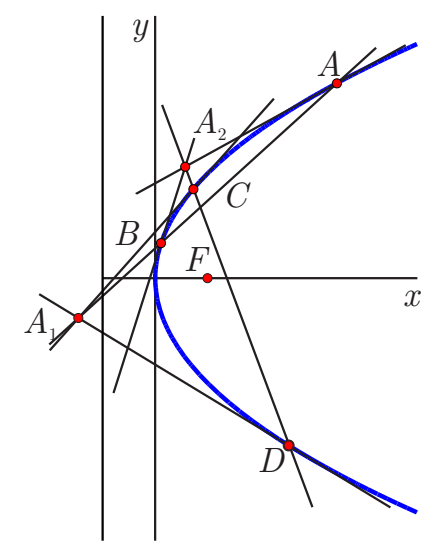

Figure 9.
Proof. Since the chord of contact from the point $A_{1}$, shown as $C D$ in the Figure, passes through $A_{2}$ by (3), we have

$2 \lambda x_{2}-y_{1} y_{2}+2 \lambda x_{1}=0$.

Rearranging the equation shows that $A_{1} \equiv\left[x_{1}, y_{1}\right]$ lies on

$\left(2 \lambda:-y_{2}: 2 \lambda x_{2}\right)$

which is the chord of contact from the point $A_{2}$, shown as $A B$ in the Figure.

\section{Quadrance properties of a parabola}

Theorem 17 Let A lie on a parabola with focus $F$. Let $T$ be the point where the tangent at $A$ meets the axis of the parabola. Then $Q(A, F)=Q(F, T)$.

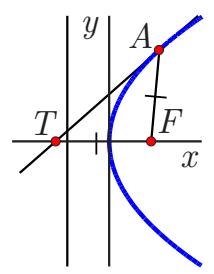

Figure 10.

Proof. Let $A \equiv\left[\lambda a^{2}, 2 \lambda a\right]$. By (2) the tangent at $A$ meets the axis at the point

$T=\left[-\lambda a^{2}, 0\right]$.

Now

$Q(A, F)=\left(\lambda a^{2}-\lambda\right)^{2}+4 \lambda^{2} a^{2}=\lambda^{2}\left(a^{2}+1\right)^{2}$

and

$Q(F, T)=\left(\lambda+\lambda a^{2}\right)^{2}=\lambda^{2}\left(a^{2}+1\right)^{2}$.

Hence $Q(A, F)=Q(F, T)$.

Theorem 18 Let $A B$ be a chord of the parabola prom the external point $X$. Let $F$ be the focus of $p$. Then

$Q(X, F)^{2}=Q(A, F) Q(F, B)$.

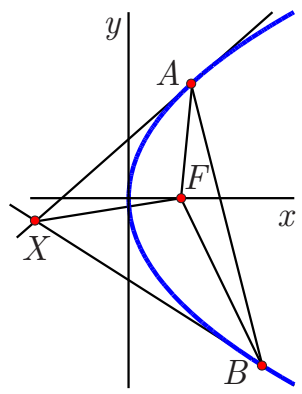

Figure 11. 
Proof. Let $A \equiv\left[\lambda a^{2}, 2 \lambda a\right]$ and $B \equiv\left[\lambda b^{2}, 2 \lambda b\right]$. By (5) the external point of the chord $A B$ is $X=[\lambda a b, \lambda(a+b)]$. Now

$$
\begin{aligned}
Q(X, F)^{2} & =\left(\lambda^{2}(a b-1)^{2}+\lambda^{2}(a+b)^{2}\right)^{2} \\
& =\lambda^{4}\left(a^{2}+b^{2}+a^{2} b^{2}+1\right)^{2} \\
& =\lambda^{4}\left(\left(a^{2}+1\right)\left(b^{2}+1\right)\right)^{2} .
\end{aligned}
$$

Moreover

$Q(A, F)=\left(\lambda a^{2}-\lambda\right)^{2}+4 \lambda^{2} a^{2}=\lambda^{2}\left(a^{2}+1\right)^{2}$.

Similarly

$Q(F, B)=\lambda^{2}\left(b^{2}+1\right)^{2}$.

Hence $Q(X, F)^{2}=Q(A, F) Q(F, B)$.

Theorem 19 If a focal chord $A B$ of a parabola with focus $F$ meets the directrix at $D$, then

$Q(A, F): Q(F, B)=Q(A, D): Q(D, B)$.

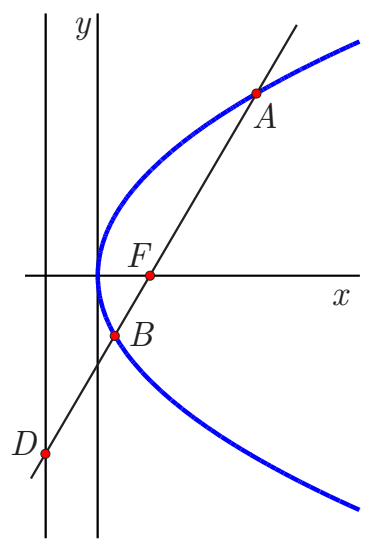

Figure 12.

Proof. Let $A \equiv\left[\lambda a^{2}, 2 \lambda a\right]$ and $B \equiv\left[\lambda b^{2}, 2 \lambda b\right]$. Then

$$
Q(A, F)=\left(\lambda a^{2}-\lambda\right)^{2}+4 \lambda^{2} a^{2}=\lambda^{2}\left(a^{2}+1\right)^{2}
$$$$
Q(F, B)=\left(\lambda b^{2}-\lambda\right)^{2}+4 \lambda^{2} b^{2}=\lambda^{2}\left(b^{2}+1\right)^{2} .
$$

Therefore

$Q(A, F): Q(F, B)=\left(1+a^{2}\right)^{2}:\left(1+b^{2}\right)^{2}$

By (4) the chord $A B$ intersects the directrix at

$D=\left[-\lambda, \frac{2 \lambda(a b-1)}{a+b}\right]$

where $a \neq-b$. Therefore

$$
\begin{aligned}
Q(A, D) & =\left(\lambda a^{2}+\lambda\right)^{2}+\left(2 \lambda a-\frac{2 \lambda(a b-1)}{a+b}\right)^{2} \\
& =\lambda^{2}\left(a^{2}+1\right)^{2}\left(1+\frac{4}{(a+b)^{2}}\right) .
\end{aligned}
$$

Similarly

$Q(D, B)=\lambda^{2}\left(b^{2}+1\right)^{2}\left(1+\frac{4}{(a+b)^{2}}\right)$.

Hence

$Q(A, F): Q(F, B)=Q(A, D): Q(D, B)$.

Theorem 20 Let $A$ and $B$ be two non-null points on $a$ parabola with focus $F$. If $A B$ is a focal chord and the tangent at $A$ meets the latus rectum at $C$. Then

$Q(C, F)^{2}=Q(A, F) Q(F, B)$.

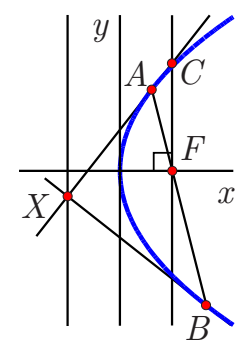

Figure 13.

Proof. Let $A \equiv\left[\lambda a^{2}, 2 \lambda a\right]$. The latus rectum is $(1: 0:-\lambda)$

which intersects the tangent at $A$

$\left(1:-a: \lambda a^{2}\right)$

at

$C \equiv\left[\lambda, \frac{\lambda\left(1+a^{2}\right)}{a}\right]$

Now

$Q(C, F)^{2}=\left(\left(\frac{\lambda\left(1+a^{2}\right)}{a}\right)^{2}\right)^{2}=\frac{\lambda^{4}\left(1+a^{2}\right)^{4}}{a^{4}}$

Moreover

$Q(A, F)=\left(\lambda a^{2}-\lambda\right)^{2}+4 \lambda^{2} a^{2}=\lambda^{2}\left(a^{2}+1\right)^{2}$.

Similarly with $B \equiv\left[\lambda b^{2}, 2 \lambda b\right]$,

$Q(F, B)=\lambda^{2}\left(b^{2}+1\right)^{2}$.

Since $A B$ is a focal chord, it passes through $F$. By (4) $a b=-1$. Therefore

$$
\begin{aligned}
Q(A, F) Q(F, B) & =\lambda^{2}\left(a^{2}+1\right)^{2} \lambda^{2}\left(b^{2}+1\right)^{2} \\
& =\lambda^{4}\left(a^{2}+1\right)^{2}\left(\frac{1}{a^{2}}+1\right)^{2} \\
& =\lambda^{4}\left(a^{2}+1\right)^{2}\left(\frac{1+a^{2}}{a^{2}}\right)^{2} \\
& =\frac{\lambda^{4}\left(a^{2}+1\right)^{4}}{a^{4}} .
\end{aligned}
$$


Hence

$Q(C, F)^{2}=Q(A, F) Q(F, B)$.

\section{Spread properties of a parabola}

Theorem 21 Let A lie on a parabola with focus $F$. Suppose the tangent at $A$ meets the directrix at D. Then AF is perpendicular to $D F$.

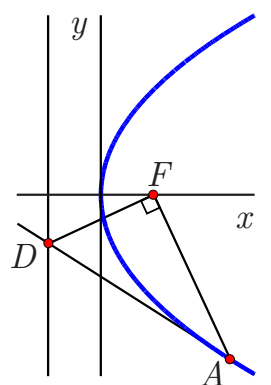

Figure 14.

Proof. Let $A \equiv\left[\lambda a^{2}, 2 \lambda a\right]$. From (2) the tangent at $A$ meets the directrix at

$D=\left[-\lambda, \frac{\lambda\left(a^{2}-1\right)}{a}\right]$

where $a \neq 0$. Now the line $A F$ is

$\left(2 a: 1-a^{2}:-2 \lambda a\right)$

and the line $D F$

$\left(a^{2}-1: 2 a:-\lambda\left(a^{2}-1\right)\right)$.

So $A F$ is perpendicular to $D F$.

Theorem 22 Let A be a non-null point on a parabola with focus $F$. Then the spread between the line AF and the axis of the parabola is a square number.

Proof. Let $A \equiv\left[\lambda a^{2}, 2 \lambda a\right]$. Then the line $A F$ has equation $\left(2 a: 1-a^{2}:-2 \lambda a\right)$.

The axis of the parabola is $(0: 1: 0)$. The spread between these two lines is

$\frac{(2 a)^{2}}{(2 a)^{2}+\left(1-a^{2}\right)^{2}}=\left(\frac{2 a}{1+a^{2}}\right)^{2}$

which is a square.

Theorem 23 Let $A$ and $B$ be two non-null points on $a$ parabola with focus $F$. Then $\overline{A B}$ subtends a square spread at the focus.
Proof. Let $A \equiv\left[\lambda a^{2}, 2 \lambda a\right], B \equiv\left[\lambda b^{2}, 2 \lambda b\right]$ Then the lines $A F$ and $B F$ are

$$
\left(2 a: 1-a^{2}:-2 \lambda a\right) \text { and }\left(2 b: 1-b^{2}:-2 \lambda b\right)
$$

respectively. Now the spread between $A F$ and $B F$ is

$$
\begin{aligned}
s(A F, B F) & =\frac{\left(2 a\left(1-b^{2}\right)-2 b\left(1-a^{2}\right)\right)^{2}}{\left((2 a)^{2}+\left(1-a^{2}\right)^{2}\right)\left((2 b)^{2}+\left(1-b^{2}\right)^{2}\right)} \\
& =\frac{(2(a-b)(1+a b))^{2}}{\left(1+a^{2}\right)^{2}\left(1+b^{2}\right)^{2}}
\end{aligned}
$$

which is a square.

Theorem 24 Let $A$ and $B$ be two points on a parabola with focus $F$ with at least one of the points non-null. Let $X$ be the external point of the chord $A B$, and suppose the line $A B$ meets the directrix of the parabola at $D$. Then $D F$ is perpendicular to $F X$.

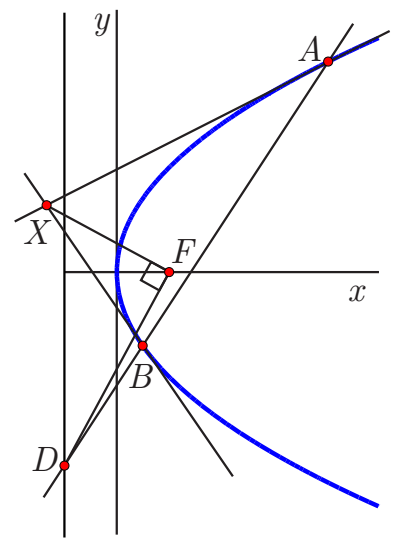

Figure 15.

Proof. With $A$ and $B$ the usual points, by (4) the line $A B$ intersects the directrix at

$D=\left[-\lambda, \frac{2 \lambda(a b-1)}{a+b}\right]$

where $a \neq-b$. By (5)

$X=[\lambda a b, \lambda(a+b)]$

Now the line $D F$ is

$(a b-1: a+b:-\lambda(a b-1))$

and the line $F X$ is

$(a+b: 1-a b:-\lambda(a+b))$.

Thus $D F$ is perpendicular to $F X$.

Theorem 25 Let $A$ and $B$ be two non-null points lying on a parabola with focus $F$. Let the tangents at $A$ and $B$ intersect the directrix at $C$ and $D$ respectively. Then $\overline{A B}$ and $\overline{C D}$ subtend equal spreads at the focus. 


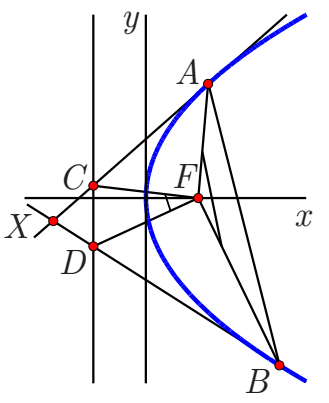

Figure 16.

Proof. By (2) the tangents at the usual points $A$ and $B$ are

$$
\left(1:-a: \lambda a^{2}\right) \quad \text { and } \quad\left(1:-b: \lambda b^{2}\right)
$$

respectively. They intersect the directrix at

$C \equiv\left[-\lambda, \frac{\lambda\left(a^{2}-1\right)}{a}\right]$ and $D \equiv\left[-\lambda, \frac{\lambda\left(b^{2}-1\right)}{b}\right]$

respectively. The line $A F$ is

$\left(2 a: 1-a^{2}:-2 \lambda a\right)$.

Similarly the line $B F$ is $\left(2 b: 1-b^{2}:-2 \lambda b\right)$. Thus

$$
\begin{aligned}
s(A F, B F) & =\frac{\left(2 a\left(1-b^{2}\right)-2 b\left(1-a^{2}\right)\right)^{2}}{\left(4 a^{2}+\left(1-a^{2}\right)^{2}\right)\left(4 b^{2}+\left(1-b^{2}\right)^{2}\right)} \\
& =\frac{4((a-b)(a b+1))^{2}}{\left(1+a^{2}\right)^{2}\left(1+b^{2}\right)^{2}} .
\end{aligned}
$$

The line $C F$ is $\left(a^{2}-1: 2 a:-\lambda\left(a^{2}-1\right)\right)$ and similarly the line $D F$ is $\left(b^{2}-1: 2 b:-\lambda\left(b^{2}-1\right)\right)$. Thus

$$
\begin{aligned}
s(C F, D F) & =\frac{\left(2 b\left(a^{2}-1\right)-2 a\left(b^{2}-1\right)\right)^{2}}{\left(\left(a^{2}-1\right)^{2}+4 a^{2}\right)\left(\left(b^{2}-1\right)^{2}+4 b^{2}\right)} \\
& =\frac{4((a-b)(1+a b))^{2}}{\left(1+a^{2}\right)^{2}\left(1+b^{2}\right)^{2}} .
\end{aligned}
$$

Hence $s(A F, B F)=s(C F, D F)$.

Theorem 26 Suppose $A$ and $B$ are two non-null points on a parabola. If $\overline{A B}$ is a side of the parabola with the external point $X$, then the sides $\overline{A X}$ and $\overline{B X}$ subtend equal spreads at the focus.

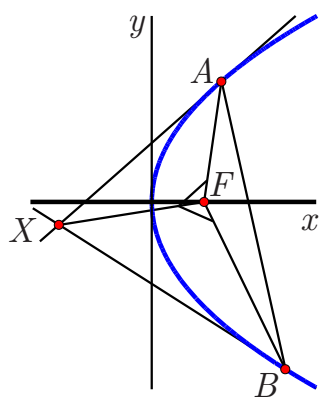

Figure 17.
Proof. Let $A \equiv\left[\lambda a^{2}, 2 \lambda a\right]$ and $B \equiv\left[\lambda b^{2}, 2 \lambda b\right]$. By (5) the tangents intersect at

$X=[\lambda a b, \lambda(a+b)]$

Then

$A F=\left(2 a: 1-a^{2}:-2 \lambda a\right)$
$F B=\left(2 b: 1-b^{2}:-2 \lambda b\right)$
$X F=(a+b: 1-a b:-\lambda(a+b))$.

Therefore

$$
\begin{aligned}
s(A F, F X) & =\frac{\left(2 a(1-a b)-\left(1-a^{2}\right)(a+b)\right)^{2}}{\left(4 a^{2}+\left(1-a^{2}\right)^{2}\right)\left((a+b)^{2}+(1-a b)^{2}\right)} \\
& =\frac{\left(a-a^{2} b+a^{3}-b\right)^{2}}{\left(1+a^{2}\right)^{2}\left(a^{2}+b^{2}+a^{2} b^{2}+1\right)} \\
& =\frac{\left((a-b)\left(1+a^{2}\right)\right)^{2}}{\left(1+a^{2}\right)^{2}\left(1+a^{2}\right)\left(1+b^{2}\right)} \\
& =\frac{(a-b)^{2}}{\left(1+a^{2}\right)\left(1+b^{2}\right)} .
\end{aligned}
$$

Since this expression is symmetrical in $a$ and $b$,

$s(A F, F X)=s(X F, F B)$.

Theorem 27 Suppose the non-null tangent at $A$ to $a$ parabola with focus $F$ passes through an external point $X$ to a parabola. Then the spread between the tangent to the parabola at $A$ and its axis is equal to the spread between the other non-null tangent and line $X F$.

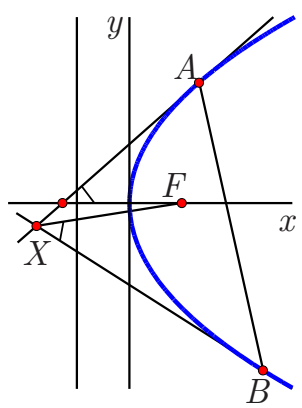

Figure 18.

Proof. Let $A \equiv\left[\lambda a^{2}, 2 \lambda a\right]$ and $B \equiv\left[\lambda b^{2}, 2 \lambda b\right]$ lie on the parabola. By (5) the tangents intersect at

$X=[\lambda a b, \lambda(a+b)]$

The axis of the parabola is

$n \equiv(0: 1: 0)$ 
Then by (2)

$A X=\left(1:-a: \lambda a^{2}\right) \quad$ and $\quad B X=\left(1:-b: \lambda b^{2}\right)$.

The equation of the line through $X$ and the focus $F$ is

$X F=(a+b: 1-a b:-\lambda(a+b))$.

Therefore

$s(A X, n)=\frac{1}{1+a^{2}}$.

Moreover

$$
\begin{aligned}
s(B X, X F) & =\frac{((1-a b)+b(a+b))^{2}}{\left(1+b^{2}\right)\left((a+b)^{2}+(1-a b)^{2}\right)} \\
& =\frac{\left(1+b^{2}\right)^{2}}{\left(1+b^{2}\right)\left(a^{2}+b^{2}+a^{2} b^{2}+1\right)} \\
& =\frac{\left(1+b^{2}\right)^{2}}{\left(1+b^{2}\right)\left(1+a^{2}\right)\left(1+b^{2}\right)} \\
& =\frac{1}{1+a^{2}} .
\end{aligned}
$$

Hence $s(A X, n)=s(B X, X F)$.

Theorem 28 Let $A$ and $B$ be two non-null points on a parabola with focus $F$. Let the tangents at $A$ and $B$ intersect at $X$. Let the spread subtended by the side $\overline{A B}$ at $X$ be $s$. Then the spread subtended by $\overline{A B}$ at the focus $F$ is equal to $S_{2}(s)=4 s(1-s)$.

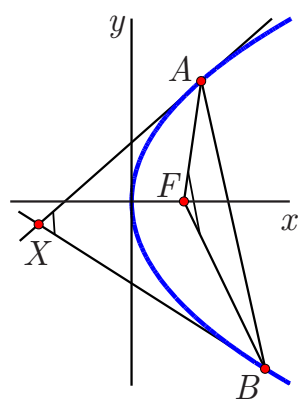

Figure 19.

Proof. Suppose $A \equiv\left[\lambda a^{2}, 2 \lambda a\right]$ and $B \equiv\left[\lambda b^{2}, 2 \lambda b\right]$ are points on the parabola. By (5) the tangents intersect at

$X=[\lambda a b, \lambda(a+b)]$.

The spread between the tangents is

$s \equiv \frac{(a-b)^{2}}{\left(1+a^{2}\right)\left(1+b^{2}\right)}$.
Therefore

$$
\begin{aligned}
S_{2}(s) & =4 s(1-s) \\
& =4 \frac{(a-b)^{2}}{\left(1+a^{2}\right)\left(1+b^{2}\right)}\left(1-\frac{(a-b)^{2}}{\left(1+a^{2}\right)\left(1+b^{2}\right)}\right) \\
& =\frac{4(a-b)^{2}}{\left(1+a^{2}\right)\left(1+b^{2}\right)}\left(\frac{1+a^{2} b^{2}+2 a b}{\left(1+a^{2}\right)\left(1+b^{2}\right)}\right) \\
& =\frac{4(a-b)^{2}(1+a b)^{2}}{\left(1+a^{2}\right)^{2}\left(1+b^{2}\right)^{2}} .
\end{aligned}
$$

The lines $A F$ and $F B$ are

$$
\left(2 a: 1-a^{2}:-2 \lambda a\right) \quad\left(2 b: 1-b^{2}:-2 \lambda b\right)
$$

respectively. Therefore

$$
\begin{aligned}
& s(A F, F B)=\frac{\left(2 a\left(1-b^{2}\right)-2 b\left(1-a^{2}\right)\right)^{2}}{\left(4 a^{2}+\left(1-a^{2}\right)^{2}\right)\left(4 b^{2}+\left(1-b^{2}\right)^{2}\right)} \\
& =\frac{4((a-b)(1+a b))^{2}}{\left(1+a^{2}\right)^{2}\left(1+b^{2}\right)^{2}} \text {. }
\end{aligned}
$$

Theorem 29 Suppose that two congruent parabolas have the same vertex, and that their axes are perpendicular to each other. If the characteristic of the field is not 5 , then the parabolas intersect with a spread of $9 / 25$. Otherwise the two parabolas intersect at one of the null points on the two parabolas.

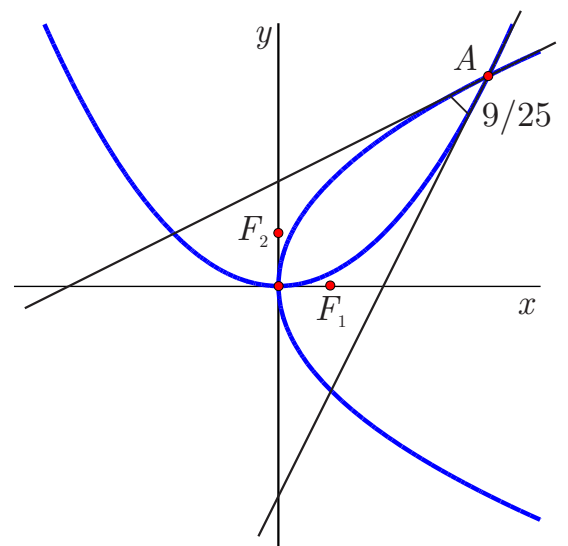

Figure 20.

Proof. Let the two congruent parabolas be

$y^{2}=4 \lambda x \quad$ and $\quad x^{2}=4 \lambda y$

with foci $F_{1}$ and $F_{2}$ respectively.

Suppose first that the two parabolas are defined over a field of characteristic other than 5. Then they intersect at

$A \equiv[4 \lambda, 4 \lambda]$.

The tangents at $A$ in (7) are

$(1:-2: 4 \lambda) \quad$ and $\quad(2:-1:-4 \lambda)$ 
respectively. Hence their spread is

$$
\frac{((1)(-1)-(2)(-2))^{2}}{\left(1^{2}+(-2)^{2}\right)\left(2^{2}+(-1)^{2}\right)}=\frac{3^{2}}{5^{2}}=\frac{9}{25} \text {. }
$$

On the other hand, if the characteristic of the field is 5 , then

$Q\left(A, F_{1}\right)=(4 \lambda-\lambda)^{2}+(4 \lambda)^{2}=25 \lambda^{2}=0$

$Q\left(A, F_{2}\right)=(4 \lambda)^{2}+(4 \lambda-\lambda)^{2}=25 \lambda^{2}=0$

which shows that the point $A$ is a null point on the parabolas.

Theorem 30 Suppose that two congruent parabolas have a common focus but different vertices. If their axes are the same, then the parabolas intersect perpendicularly.

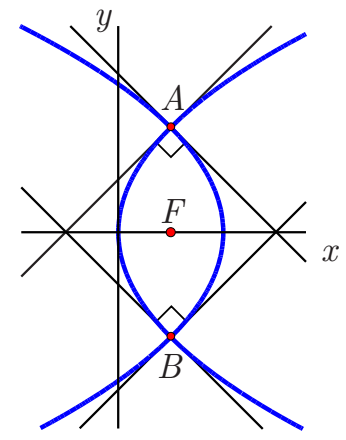

Figure 21.

Proof. Let the parabolas be

$y^{2}=4 \lambda x \quad$ and $\quad y^{2}=-4 \lambda(x-2 \lambda)$.

They intersect at

$A=[\lambda, 2 \lambda] \quad$ and $\quad B=[\lambda,-2 \lambda]$.

The tangents at $A$ for (8) are

$(1:-1: \lambda) \quad$ and $\quad(1: 1:-3 \lambda)$

respectively, which are perpendicular. Similarly the tangents at $B$ in (8) are

$(1: 1: \lambda) \quad$ and $\quad(1:-1:-3 \lambda)$

respectively, which are also perpendicular.

\section{Signed areas and anti-symmetric polyno- mials}

In this section we review some definitions, notation and results from [16] which we quickly review. If $A_{1} \equiv\left[x_{1}, y_{1}\right]$,
$A_{2} \equiv\left[x_{2}, y_{2}\right]$ and $A_{3} \equiv\left[x_{3}, y_{3}\right]$, then the signed area of the oriented triangle $\overrightarrow{A_{1} A_{2} A_{3}}$ is the number

$$
\begin{aligned}
a\left(\overrightarrow{A_{1} A_{2} A_{3}}\right) & \equiv \frac{x_{1} y_{2}-x_{2} y_{1}+x_{2} y_{3}-x_{3} y_{2}+x_{3} y_{1}-x_{1} y_{3}}{2} \\
& =\frac{1}{2}\left|\begin{array}{lll}
x_{1} & y_{1} & 1 \\
x_{2} & y_{2} & 1 \\
x_{3} & y_{3} & 1
\end{array}\right| .
\end{aligned}
$$

This concept is extended to more general $n$-gons, by for example defining the signed area of an oriented quadrilateral $\overrightarrow{A_{1} A_{2} A_{3} A_{4}}$ to be

$a\left(\overrightarrow{A_{1} A_{2} A_{3} A_{4}}\right)=a\left(\overrightarrow{A_{1} A_{2} A_{3}}\right)+a\left(\overrightarrow{A_{1} A_{3} A_{4}}\right)$.

To deal with a wide variety of anti-symmetric expressions in the variable $x_{i}$ and $y_{j}$ for $i, j=1,2$ and 3 , introduce the following notation. For any polynomial $r$ in these variables, define $[r]_{3}^{-}$to be the alternating sum of the six terms obtained from $r$ by applying the following changes to the indices:

$$
2 \longleftrightarrow 3 \quad 1 \longleftrightarrow 2 \quad 2 \longleftrightarrow 3 \quad 1 \longleftrightarrow 2 \quad 2 \longleftrightarrow 3 .
$$

in this order. The expression in the numerator of $a\left(\overrightarrow{A_{1} A_{2} A_{3}}\right)$ is $\left[x_{1} y_{2}\right]_{3}^{-}$. Here is another example:

$\left[x_{1} x_{2}^{3} y_{2}\right]_{3}^{-}=x_{1} x_{2}^{3} y_{2}-x_{1} x_{3}^{3} y_{3}+x_{2} x_{3}^{3} y_{3}-x_{3} x_{2}^{3} y_{2}+x_{3} x_{1}^{3} y_{1}-x_{2} x_{1}^{3} y_{1}$.

Theorem 31 (Circumcenter formula) If $A_{1} \equiv\left[x_{1}, y_{1}\right]$, $A_{2} \equiv\left[x_{2}, y_{2}\right]$ and $A_{3} \equiv\left[x_{3}, y_{3}\right]$, then the circumcenter $C$ of the triangle $\overline{A_{1} A_{2} A_{3}}$ is

$C=\left[\frac{\left[x_{1}^{2} y_{2}\right]_{3}^{-}+\left[y_{1}^{2} y_{2}\right]_{3}^{-}}{2\left[x_{1} y_{2}\right]_{3}^{-}}, \frac{\left[x_{1} x_{2}^{2}\right]_{3}^{-}+\left[x_{1} y_{2}^{2}\right]_{3}^{-}}{2\left[x_{1} y_{2}\right]_{3}^{-}}\right]$.

Theorem 32 (Orthocentre formula) If $A_{1} \equiv\left[x_{1}, y_{1}\right]$, $A_{2} \equiv\left[x_{2}, y_{2}\right]$ and $A_{3} \equiv\left[x_{3}, y_{3}\right]$, then the orthocentre $O$ of the triangle $\overline{A_{1} A_{2} A_{3}}$ is

$O=\left[\frac{\left[x_{1} x_{2} y_{2}\right]_{3}^{-}+\left[y_{1} y_{2}^{2}\right]_{3}^{-}}{\left[x_{1} y_{2}\right]_{3}^{-}}, \frac{\left[x_{1} y_{1} y_{2}\right]_{3}^{-}+\left[x_{1}^{2} x_{2}\right]_{3}^{-}}{\left[x_{1} y_{2}\right]_{3}^{-}}\right]$.

We generally use determinants to evaluate anti-symmetric expressions.

\section{Parabolic triangles}

If all three points lie on the parabola, then we have a parabolic triangle.

Theorem 33 Let $A, B$ and $C$ be three distinct points on a parabola. Let the external points of the sides $\overline{A B}, \overline{B C}$ and $\overline{C A}$ be $X, Y$ and $Z$ respectively. Then

$a(\overrightarrow{A B C}): a(\overrightarrow{X Y Z})=-2: 1$ 


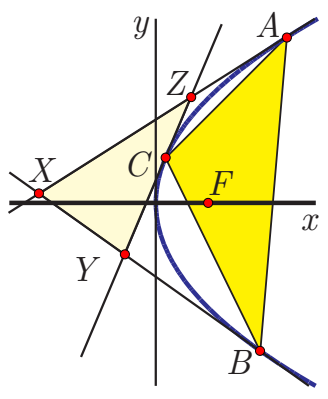

Figure 22.

Proof. Let the points on the parabola be

$$
A \equiv\left[\lambda a^{2}, 2 \lambda a\right] \quad B \equiv\left[\lambda b^{2}, 2 \lambda b\right] \quad C \equiv\left[\lambda c^{2}, 2 \lambda c\right] .
$$

Then by (5) the corresponding external points to the parabola are

$X \equiv[\lambda a b, \lambda(a+b)] \quad Y \equiv[\lambda b c, \lambda(b+c)] \quad Z \equiv[\lambda c a, \lambda(c+a)]$.

Now by the signed area formula

$$
\begin{aligned}
a(\overrightarrow{A B C}) & =\frac{1}{2}\left|\begin{array}{lll}
\lambda a^{2} & 2 \lambda a & 1 \\
\lambda b^{2} & 2 \lambda b & 1 \\
\lambda c^{2} & 2 \lambda c & 1
\end{array}\right| \\
& =\lambda^{2}\left|\begin{array}{lll}
a^{2} & a & 1 \\
b^{2} & b & 1 \\
c^{2} & c & 1
\end{array}\right| \\
& =\lambda^{2}\left|\begin{array}{ccc}
(a-b)(a+b) & (a-b) & 0 \\
(b-c)(b+c) & (b-c) & 0 \\
c^{2} & c & 1
\end{array}\right| \\
& =\lambda^{2}\left|\begin{array}{ccc}
(a-b)(a+b) & (a-b) \\
(b-c)(b+c) & (b-c)
\end{array}\right| \\
& =\lambda^{2}(a-b)(b-c)\left|\begin{array}{cc}
a+b & 1 \\
b+c & 1
\end{array}\right| \\
& =\lambda^{2}(a-b)(b-c)(a-c) .
\end{aligned}
$$

Moreover

$$
\begin{aligned}
a(\overrightarrow{X Y Z}) & =\frac{1}{2}\left|\begin{array}{ccc}
\lambda a b & \lambda(a+b) & 1 \\
\lambda b c & \lambda(b+c) & 1 \\
\lambda c a & \lambda(c+a) & 1
\end{array}\right| \\
& =\frac{\lambda^{2}}{2}\left|\begin{array}{ccc}
a b & a+b & 1 \\
b c & b+c & 1 \\
c a & c+a & 1
\end{array}\right| \\
& =\frac{\lambda^{2}}{2}\left|\begin{array}{ccc}
b(a-c) & a-c & 0 \\
c(b-a) & b-a & 0 \\
c a & c+a & 1
\end{array}\right| \\
& =\frac{\lambda^{2}}{2}\left|\begin{array}{cc}
b(a-c) & a-c \\
c(b-a) & b-a
\end{array}\right| \\
& =\frac{\lambda^{2}(a-c)(b-a)\left|\begin{array}{cc}
b \\
c & 1
\end{array}\right|}{2} \mid \\
& =\frac{\lambda^{2}(a-c)(b-a)(b-c)}{2} .
\end{aligned}
$$

Hence

$a(\overrightarrow{A B C}): a(\overrightarrow{X Y Z})=-2: 1$

Corollary 1 Let $A_{1}, A_{2}, \ldots, A_{n}$ be $n$ distinct points on a parabola. Let the external points of the sides $\overline{A_{1} A_{2}}, \overline{A_{2} A_{3}}$, $\ldots, \overline{A_{n} A_{1}}$ be $X_{1}, X_{2}, \ldots, X_{n}$ respectively. Then from the previous theorem

$a\left(\overrightarrow{A_{1} A_{2} \cdots A_{n}}\right): a\left(\overrightarrow{X_{1} X_{2} \cdots X_{n}}\right)=-2: 1$.

Proof. Let the external points of the chords $\overline{A_{1} A_{3}}, \overline{A_{1} A_{4}}$, $\ldots, \overline{A_{1} A_{n-1}}$ be $Y_{3}, Y_{4}, \ldots, Y_{n-1}$ respectively. Then

$$
\begin{aligned}
a( & \left.\overrightarrow{A_{1} A_{2} \cdots A_{n}}\right) \\
= & a\left(\overrightarrow{A_{1} A_{2} A_{3}}\right)+a\left(\overrightarrow{A_{1} A_{3} A_{4}}\right)+\cdots+a\left(\overrightarrow{A_{1} A_{k} A_{k+1}}\right)+\cdots \\
& +a\left(\overrightarrow{A_{1} A_{n-1} A_{n}}\right) \\
= & -2\left(a\left(\overrightarrow{X_{1} X_{2} Y_{3}}\right)+a\left(\overrightarrow{Y_{3} X_{3} Y_{4}}\right)+\cdots+a\left(\overrightarrow{Y_{k} X_{k} Y_{k+1}}\right)+\cdots\right. \\
& \left.+a\left(\overrightarrow{Y_{n-1} X_{n-1} X_{n}}\right)\right) \\
= & -2\left(a\left(\overrightarrow{X_{1} X_{2} Y_{3}}\right)+a\left(\overrightarrow{X_{3} Y_{4} Y_{3}}\right)+\cdots+a\left(\overrightarrow{X_{k} Y_{k+1} Y_{k}}\right)+\cdots\right. \\
& \left.+a\left(\overrightarrow{X_{n-1} X_{n} Y_{n-1}}\right)\right) \\
= & -2\left(a\left(\overrightarrow{X_{1} X_{2} X_{3} Y_{4}}\right)+\cdots+a\left(\overrightarrow{X_{k} Y_{k+1} Y_{k}}\right)+\cdots\right. \\
& \left.+a\left(\overrightarrow{X_{n-1} X_{n} Y_{n-1}}\right)\right) \\
= & -2\left(a\left(\overrightarrow{X_{1} X_{2} \cdots X_{k} Y_{k+1}}\right)+\cdots+a\left(\overrightarrow{X_{n-1} X_{n} Y_{n-1}}\right)\right) \\
= & -2 a\left(\overrightarrow{X_{1} X_{2} \cdots X_{n-1} Y_{n}}\right) .
\end{aligned}
$$

Hence $a\left(\overrightarrow{A_{1} A_{2} \cdots A_{n}}\right): a\left(\overrightarrow{X_{1} X_{2} \cdots X_{n}}\right)=-2: 1$.

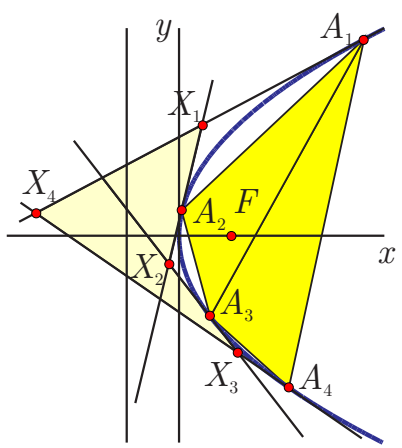

Figure 23.

The following is in [15].

Theorem 34 Let $A, B$ and $C$ be three distinct points on a parabola. Let the external points of the sides $\overline{A B}, \overline{B C}$ and $\overline{C A}$ be $X, Y$ and $Z$ respectively. Then

$\frac{Q(X, A)}{Q(A, Z)}=\frac{Q(B, X)}{Q(X, Y)}=\frac{Q(Y, Z)}{Q(Z, C)}$. 
Proof. Suppose the points on the parabola are

$$
A \equiv\left[\lambda a^{2}, 2 \lambda a\right] \quad B \equiv\left[\lambda b^{2}, 2 \lambda b\right] \quad C \equiv\left[\lambda c^{2}, 2 \lambda c\right] .
$$

Then by (5) the corresponding external points to the parabola are

$X \equiv[\lambda a b, \lambda(a+b)] \quad Y \equiv[\lambda b c, \lambda(b+c)] \quad Z \equiv[\lambda c a, \lambda(c+a)]$.

Now

$$
\begin{aligned}
Q(X, A) & =\left(\lambda a b-\lambda a^{2}\right)^{2}+(\lambda(a+b)-2 \lambda a)^{2} \\
& =\lambda^{2} a^{2}(b-a)^{2}+\lambda^{2}(b-a)^{2} \\
& =\lambda^{2}(b-a)^{2}\left(a^{2}+1\right)
\end{aligned}
$$

and

$$
\begin{aligned}
Q(A, Z) & =\left(\lambda a^{2}-\lambda c a\right)^{2}+(2 \lambda a-\lambda(c+a))^{2} \\
& =\lambda^{2} a^{2}(a-c)^{2}+\lambda^{2}(a-c)^{2} \\
& =\lambda^{2}(a-c)^{2}\left(a^{2}+1\right) .
\end{aligned}
$$

Therefore

$$
\frac{Q(X, A)}{Q(A, Z)}=\frac{(b-a)^{2}}{(a-c)^{2}} \text {. }
$$

Similarly

$$
\frac{Q(B, X)}{Q(X, Y)}=\frac{(b-a)^{2}}{(a-c)^{2}} \quad \frac{Q(Y, Z)}{Q(Z, C)}=\frac{(b-a)^{2}}{(a-c)^{2}} .
$$

Hence

$$
\frac{Q(X, A)}{Q(A, Z)}=\frac{Q(B, X)}{Q(X, Y)}=\frac{Q(Y, Z)}{Q(Z, C)} .
$$

Theorem 35 Let $A, B$ and $C$ be three distinct points on a parabola. Let the external points of the sides $\overline{A B}, \overline{B C}$ and $\overline{C A}$ be $X, Y$ and $Z$ respectively. If the parabola is defined over a field with -3 not a square, then the lines $A Y, B Z$ and $C X$ are concurrent. Otherwise if the lines $A Y, B Z$ and $C X$ are not concurrent, then they are mutually parallel to each other.

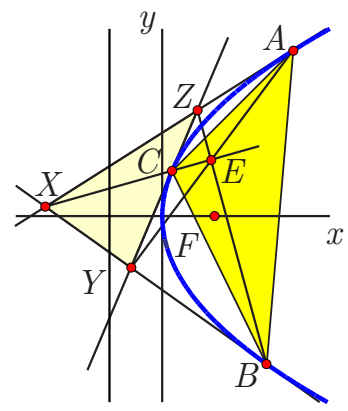

Figure 24.
Proof. Suppose the points on the parabola are $A \equiv\left[\lambda a^{2}, 2 \lambda a\right] \quad B \equiv\left[\lambda b^{2}, 2 \lambda b\right] \quad$ and $\quad C \equiv\left[\lambda c^{2}, 2 \lambda c\right]$ so that $X \equiv[\lambda a b, \lambda(a+b)] \quad Y \equiv[\lambda b c, \lambda(b+c)] \quad Z \equiv[\lambda c a, \lambda(c+a)]$. Then

$$
\begin{aligned}
& A Y=\left(2 a-(b+c): b c-a^{2}: \lambda\left(a^{2}(b+c)-2 a b c\right)\right) \\
& B Z=\left(2 b-(c+a): c a-b^{2}: \lambda\left(b^{2}(c+a)-2 a b c\right)\right) \\
& C X=\left(2 c-(a+b): a b-c^{2}: \lambda\left(c^{2}(a+b)-2 a b c\right)\right) .
\end{aligned}
$$

Suppose $A Y, B Z$ and $C X$ are mutually parallel to each other. Then

$$
\begin{aligned}
& (2 a-(b+c)) \times\left(c a-b^{2}\right)-(2 b-(c+a)) \times\left(b c-a^{2}\right) \\
& \quad=(b-a)\left(a^{2}-c a-b a+b^{2}+c^{2}-b c\right)=0 \\
& (2 a-(b+c)) \times\left(a b-c^{2}\right)-(2 c-(a+b)) \times\left(b c-a^{2}\right) \\
& \quad=(c-a)\left(a^{2}-c a-b a+b^{2}+c^{2}-b c\right)=0 .
\end{aligned}
$$

Therefore a sufficient condition for the three lines to be mutually parallel is

$a^{2}+b^{2}+c^{2}-a b-b c-c a=0$.

If we solve for $c$ in (9), then we obtain a quadratic in $c$. Its discriminant is

$-3 a^{2}+6 b a-3 b^{2}=-3(a-b)^{2}$.

Hence if -3 is a square, then without loss of generality the point $C$ on the parabola can be chosen such that $A Y, B Z$ and $C X$ are mutually parallel.

Now suppose that -3 is not a square; then the three lines are not mutually parallel. Therefore

$$
\begin{aligned}
& \left|\begin{array}{ccc}
2 a-(b+c) & b c-a^{2} & \lambda\left(a^{2}(b+c)-2 a b c\right) \\
2 b-(c+a) & c a-b^{2} & \lambda\left(b^{2}(c+a)-2 a b c\right) \\
2 c-(a+b) & a b-c^{2} & \lambda\left(c^{2}(a+b)-2 a b c\right)
\end{array}\right| \\
& =\left|\begin{array}{ccc}
3(a-b) & -(a-b)(a+b+c) & \lambda(a-b)(a b+b c+c a) \\
2 b-(c+a) & b^{2}-c a & \lambda\left(b^{2}(c+a)-2 a b c\right) \\
2 c-(a+b) & c^{2}-a b & \lambda\left(c^{2}(a+b)-2 a b c\right)
\end{array}\right| \\
& =\left|\begin{array}{ccc}
3(a-b) & -(a-b)(a+b+c) & \lambda(a-b)(a b+b c+c a) \\
3(b-c) & -(b-c)(a+b+c) & \lambda(b-c)(a b+b c+c a) \\
2 c-(a+b) & c^{2}-a b & \lambda\left(c^{2}(a+b)-2 a b c\right)
\end{array}\right| \\
& =(a-b)(b-c)\left|\begin{array}{ccc}
3 & -(a+b+c) & \lambda(a b+b c+c a) \\
3 & -(a+b+c) & \lambda(a b+b c+c a) \\
2 c-(a+b) & c^{2}-a b & \lambda\left(c^{2}(a+b)-2 a b c\right)
\end{array}\right| \\
& =0 .
\end{aligned}
$$

Therefore by the Concurrent lines theorem the lines $A Y$, $B Z$ and $C X$ are concurrent. Hence if -3 is not a square, the lines $A Y, B Z$ and $C X$ are always concurrent. Otherwise if the lines are not concurrent then the three lines must be mutually parallel.

Call the common point of intersection, which is $E$ in Figure 24 , the central point of the parabolic triangle $\overline{A B C}$. 
Theorem 36 Let $A, B$ and $C$ be three distinct points on a parabola. Let the external points of the sides $\overline{A B}, \overline{B C}$ and $\overline{C A}$ be $X, Y$ and $Z$ respectively. Then the centroids of the triangles $\overline{X A B}, \overline{Y B C}$ and $\overline{Z C A}$ are collinear. Moreover the central point of $\overline{A B C}$ lies on that line.

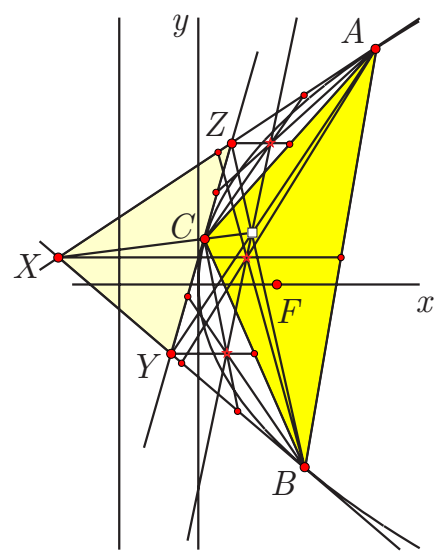

Figure 25.

Proof. Let the points on the parabola be

$A \equiv\left[\lambda a^{2}, 2 \lambda a\right] \quad B \equiv\left[\lambda b^{2}, 2 \lambda b\right]$ and $C \equiv\left[\lambda c^{2}, 2 \lambda c\right]$.

Then by (5) the external points to the parabola are

$X \equiv[\lambda a b, \lambda(a+b)] \quad Y \equiv[\lambda b c, \lambda(b+c)]$

and $Z \equiv[\lambda c a, \lambda(c+a)]$.

The centroid of triangle $\overline{X A B}$ is

$\left[\frac{\lambda\left(a^{2}+a b+b^{2}\right)}{3}, \lambda(a+b)\right]$.

Similarly the centroids of triangle $\overline{Y B C}$ and $\overline{Z C A}$ are

$\left[\frac{\lambda\left(b^{2}+b c+c^{2}\right)}{3}, \lambda(b+c)\right]$ and

$\left[\frac{\lambda\left(c^{2}+c a+a^{2}\right)}{3}, \lambda(c+a)\right]$

respectively. Therefore

$$
\begin{aligned}
& \left|\begin{array}{ccc}
\lambda\left(a^{2}+a b+b^{2}\right) / 3 & \lambda(a+b) & 1 \\
\lambda\left(b^{2}+b c+c^{2}\right) / 3 & \lambda(b+c) & 1 \\
\lambda\left(c^{2}+c a+a^{2}\right) / 3 & \lambda(c+a) & 1
\end{array}\right| \\
& =\frac{\lambda^{2}}{3}\left|\begin{array}{ccc}
a^{2}+a b+b^{2} & a+b & 1 \\
b^{2}+b c+c^{2} & b+c & 1 \\
c^{2}+c a+a^{2} & c+a & 1
\end{array}\right| \\
& =\frac{\lambda^{2}}{3}\left|\begin{array}{ccc}
(a+b+c)(a-c) & a-c & 0 \\
b^{2}+b c+c^{2} & b+c & 1 \\
c^{2}+c a+a^{2} & c+a & 1
\end{array}\right| \\
& =\frac{\lambda^{2}}{3}\left|\begin{array}{ccc}
(a+b+c)(a-c) & a-c & 0 \\
(a+b+c)(b-a) & b-a & 0 \\
c^{2}+c a+a^{2} & c+a & 1
\end{array}\right|=0 .
\end{aligned}
$$

The line containing the three centroids is

$(3:-(a+b+c): \lambda(a b+b c+c a))$.

The coordinate of the central point is

$$
\left[\begin{array}{c}
\frac{\lambda\left(a^{2}(b-c)^{2}+b^{2}(c-a)^{2}+c^{2}(a-b)^{2}\right)}{(a-b)^{2}+(b-c)^{2}+(c-a)^{2}} \\
\frac{2 \lambda\left(a(b-c)^{2}+b(c-a)^{2}+c(a-b)^{2}\right)}{(a-b)^{2}+(b-c)^{2}+(c-a)^{2}}
\end{array}\right] .
$$

Now

$$
\begin{aligned}
& 3\left(\frac{\lambda\left(a^{2}(b-c)^{2}+b^{2}(c-a)^{2}+c^{2}(a-b)^{2}\right)}{(a-b)^{2}+(b-c)^{2}+(c-a)^{2}}\right) \\
& -(a+b+c)\left(\frac{2 \lambda\left(a(b-c)^{2}+b(c-a)^{2}+c(a-b)^{2}\right)}{(a-b)^{2}+(b-c)^{2}+(c-a)^{2}}\right)
\end{aligned}
$$$$
+\lambda(a b+b c+c a)
$$$$
=\frac{\lambda\left(\begin{array}{l}
3\left(a^{2}(b-c)^{2}+b^{2}(c-a)^{2}+c^{2}(a-b)^{2}\right) \\
-2\left(a(b-c)^{2}+b(c-a)^{2}+c(a-b)^{2}\right)(a+b+c) \\
+(a b+b c+c a)\left((a-b)^{2}+(b-c)^{2}+(c-a)^{2}\right)
\end{array}\right)}{(a-b)^{2}+(b-c)^{2}+(c-a)^{2}}
$$$$
\lambda \frac{\lambda\left(\begin{array}{l}
a^{2}(b-c)^{2}+b^{2}(c-a)^{2}+c^{2}(a-b)^{2} \\
-(a b+a c)(b-c)^{2}-(a b+b c)(c-a)^{2} \\
-(a c+b c)(a-b)^{2} \\
+a b(a-b)^{2}+b c(b-c)^{2}+c a(c-a)^{2}
\end{array}\right)}{(a-b)^{2}+(b-c)^{2}+(c-a)^{2}}
$$$$
=\frac{\lambda\left(\begin{array}{c}
(c-a)(c-b)(a-b)^{2} \\
+(a-b)(a-c)(b-c)^{2} \\
+(b-c)(b-a)(c-a)^{2}
\end{array}\right)}{(a-b)^{2}+(b-c)^{2}+(c-a)^{2}}
$$$$
=\frac{\lambda(a-b)(b-c)(c-a)(-(a-b)-(b-c)-(c-a))}{(a-b)^{2}+(b-c)^{2}+(c-a)^{2}}
$$

$=0$.

Hence the central point lies on the line.

Theorem 37 Let $A, B$ and $C$ be three distinct points on a parabola. Let the external points of the sides $\overline{A B}, \overline{B C}$ and $\overline{C A}$ be $X, Y$ and $Z$ respectively. Then the orthocentre of triangle $\overline{X Y Z}$ lies on the directrix.

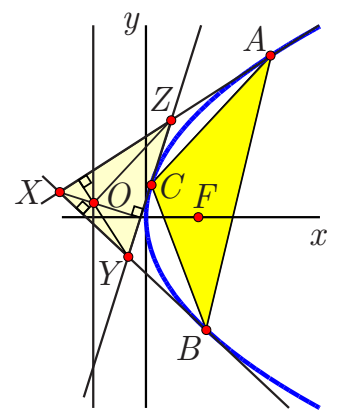

Figure 26. 
Proof. Using our standard notation,

$X \equiv[\lambda a b, \lambda(a+b)] \quad Y \equiv[\lambda b c, \lambda(b+c)]$

and $Z \equiv[\lambda c a, \lambda(c+a)]$.

Calculating at the abscissa of the orthocentre

$$
\begin{aligned}
& {\left[x_{1} x_{2} y_{2}\right]_{3}^{-}=} \lambda^{3} a b c(b-a)(a+b+c)+\lambda^{3} a b c^{2}(a-b) \\
&+\lambda^{3} a b c(a+b)(a-b) \\
&= \lambda^{3} a b c(a-b)(-(a+b+c)+c+a+b) \\
&= 0 \\
& {\left[y_{1} y_{2}^{2}\right]_{3}^{-}=\left|\begin{array}{ccc}
\lambda(a+b) & \lambda^{2}(a+b)^{2} & 1 \\
\lambda(b+c) & \lambda^{2}(b+c)^{2} & 1 \\
\lambda(c+a) & \lambda^{2}(c+a)^{2} & 1
\end{array}\right| } \\
&=\lambda^{3}\left|\begin{array}{ccc}
a-c & (a+2 b+c)(a-c) & 0 \\
b-a & (a+b+2 c)(b-a) & 0 \\
c+a & (c+a)^{2} & 1
\end{array}\right| \\
&=\lambda^{3}(a-c)(b-a)(c-b) \\
& {\left[x_{1} y_{2}\right]_{3}^{-}=} \lambda^{2}(a-b)(b-c)(c-a) .
\end{aligned}
$$

Therefore

$$
\begin{aligned}
\frac{\left[x_{1} x_{2} y_{2}\right]_{3}^{-}+\left[y_{1} y_{2}^{2}\right]_{3}^{-}}{\left[x_{1} y_{2}\right]_{3}^{-}} & =\frac{0+\lambda^{3}(a-c)(b-a)(c-b)}{\lambda^{2}(a-b)(b-c)(c-a)} \\
& =-\lambda .
\end{aligned}
$$

Hence the orthocentre lies on the directrix.

Theorem 38 Let $A, B$ and $C$ be three distinct points on a parabola. Let the external points of the sides $\overline{A B}, \overline{B C}$ and $\overline{C A}$ be $X, Y$ and $Z$ respectively. Let the orthocentres of the triangles $\overline{X A B}, \overline{Y B C}$ and $\overline{Z C A}$ be $O_{X}, O_{Y}$ and $O_{Z}$ respectively. Then

$a\left(\overrightarrow{O_{X} O_{Y} O_{Z}}\right)=-a(\overrightarrow{A B C})$.

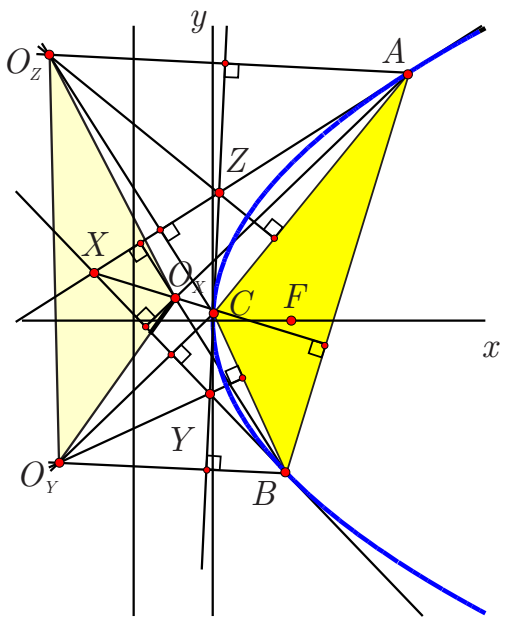

Figure 27.
Proof. Using the standard notation and the orthocentre formula

$\begin{aligned} O_{X} & =[-\lambda(2+a b), \lambda(2+a b)(a+b)] \\ O_{Y} & =[-\lambda(2+b c), \lambda(2+b c)(b+c)] \\ O_{Z} & =[-\lambda(2+c a), \lambda(2+c a)(c+a)] .\end{aligned}$

Therefore by the signed area formula

$$
\begin{aligned}
& a\left(\overrightarrow{O_{X} O_{Y} O_{Z}}\right)=\frac{1}{2}\left|\begin{array}{ccc}
-\lambda(2+a b) & \lambda(2+a b)(a+b) & 1 \\
-\lambda(2+b c) & \lambda(2+b c)(b+c) & 1 \\
-\lambda(2+c a) & \lambda(2+c a)(c+a) & 1
\end{array}\right| \\
& =-\frac{\lambda^{2}}{2}\left|\begin{array}{ccc}
2+a b & (2+a b)(a+b) & 1 \\
2+b c & (2+b c)(b+c) & 1 \\
2+c a & (2+c a)(c+a) & 1
\end{array}\right| \\
& \quad=-\frac{\lambda^{2}}{2}\left|\begin{array}{ccc}
b(a-c) & (a-c)\left(b^{2}+b(a+c)+2\right) & 0 \\
c(b-a) & (b-a)\left(c^{2}+c(b+a)+2\right) & 0 \\
2+c a & (2+c a)(c+a) & 1
\end{array}\right| \\
& =-\frac{\lambda^{2}(a-c)(b-a)}{2}\left|\begin{array}{cc}
b & b^{2}+b(a+c)+2 \\
c & c^{2}+c(b+a)+2
\end{array}\right| \\
& =\lambda^{2}(a-b)(b-c)(c-a)
\end{aligned}
$$

But from the proof of Theorem 34,

$a(\overrightarrow{A B C})=\lambda^{2}(a-b)(b-c)(a-c)$.

Hence $a\left(\overrightarrow{O_{X} O_{Y} O_{Z}}\right)=-a(\overrightarrow{A B C})$.

In the remaining theorem, we continue with our established notation. Recall that triangles are similar precisely when corresponding spreads are equal.

Theorem 39 Let the circumcentres of the triangles $\overline{X A B}$, $\overline{Y B C}$ and $\overline{Z C A}$ be $C_{X}, C_{Y}$ and $C_{Z}$ respectively. Then triangle $\overline{C_{X} C_{Y} C_{Z}}$ is similar to triangle $\overline{X Y Z}$.

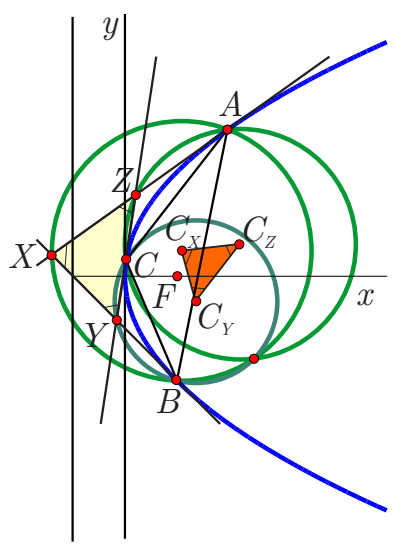

Figure 28. 
Proof. (Using a computer) By the circumcenter formula, and using a computer,

$\begin{aligned} C_{X} & =\left[\frac{\lambda\left(a^{2}+2 a b+b^{2}+2\right)}{2},-\frac{\lambda(a+b)(a b-1)}{2}\right] \\ C_{Y} & =\left[\frac{\lambda\left(b^{2}+2 b c+c^{2}+2\right)}{2},-\frac{\lambda(b+c)(b c-1)}{2}\right] \\ C_{Z} & =\left[\frac{\lambda\left(a^{2}+2 c a+c^{2}+2\right)}{2},-\frac{\lambda(c+a)(c a-1)}{2}\right] .\end{aligned}$

We may now calculate the spreads

$s\left(C_{Y} C_{X}, C_{X} C_{Z}\right)=\frac{(a-b)^{2}}{\left(a^{2}+1\right)\left(b^{2}+1\right)}$

$s\left(C_{Z} C_{Y}, C_{Y} C_{X}\right)=\frac{(b-c)^{2}}{\left(b^{2}+1\right)\left(c^{2}+1\right)}$

$s\left(C_{X} C_{Z}, C_{Z} C_{Y}\right)=\frac{(a-c)^{2}}{\left(c^{2}+1\right)\left(a^{2}+1\right)}$.

Similarly in triangle $\overline{A B C}$ we find that

$s(Y X, X Z)=\frac{(a-b)^{2}}{\left(a^{2}+1\right)\left(b^{2}+1\right)}$

$s(Z Y, Y Z)=\frac{(b-c)^{2}}{\left(b^{2}+1\right)\left(c^{2}+1\right)}$

$s(X Z, Z Y)=\frac{(a-c)^{2}}{\left(c^{2}+1\right)\left(a^{2}+1\right)}$.

Therefore

$s\left(C_{Y} C_{X}, C_{X} C_{Z}\right)=s(Y X, X Z)$
$s\left(C_{Z} C_{Y}, C_{Y} C_{X}\right)=s(Z Y, Y Z)$
$s\left(C_{X} C_{Z}, C_{Z} C_{Y}\right)=s(X Z, Z Y)$.

Thus triangles $\overline{C_{X} C_{Y} C_{Z}}$ and $\overline{A B C}$ have identical spreads. Hence triangles $\overline{C_{X} C_{Y} C_{Z}}$ and $\overline{A B C}$ are similar.

\section{References}

[1] A. Alkhaldi, N.J. Wildberger, The Parabola in Universal Hyperbolic Geometry I, KoG 17 (2013), 14-41.

[2] A. Alkhaldi, N.J. Wildberger, The Parabola in Universal Hyperbolic Geometry I, J. Geom. Graph. 20 (2016), 1-11.

[3] L.P. Eisenhart, Coordinate Geometry, Dover Publication Inc., Yew York, 1960.
[4] E.M. Hartley, Cartesian Geometry of the Plane, Cambridge University Press, 1966.

[5] R. Honsberger, Episodes in Nineteenth and Twentieth Century Euclidean Geometry, The Mathematical Association of America, 1995.

[6] E.A. MAXWELL, Elementary Coordinate Geometry (2nd ed.), Oxford at the Clarendon Press, 1958.

[7] W.K. Morrill, Analytic Geometry, The Haddon Craftsmen Inc. Scranton, 1951.

[8] W.F. Osgood, Plane and Solid Analytic Geometry, The Macmillan Company, New York, 1956.

[9] W. Pender, Cambridge 3 Unit Mathematics Year 11, Cambridge University Press, 1999.

[10] A. Robson, An Introduction to Analytical Geometry Volume I, Cambridge University Press, 1949.

[11] G. Salmon, A Treatise on Conic Sections (6th ed.),Longmans, Green, and Co., London, 1879.

[12] D.M.Y. Sommerville, Analytical Conics, G. Bell, London, 1933.

[13] B. Spain, Analytical conics, Pergamon Press, London, 1957.

[14] I. Todhunter, A Treatise on Plane Co-ordinate Geometry as applied to the straight line and the conic sections (4th ed.), Macmillan, London, 1867.

[15] D. Wells, The Penguin Dictionary of Curious and Interesting Geometry, Penguin, London, 1991.

[16] N.J. Wildberger, Divine Proportions: Rational Trigonometry to Universal Geometry, Wild Egg Books, Sydney, 2005.

\section{Si Chun Choi}

orcid.org/0000-0003-3856-0751

e-mail: si.choi@det.nsw.edu.au

Caringbah High School

Sydney, Australia

N.J. Wildberger

orcid.org/0000-0003-3503-6495

e-mail: n.wildberger@unsw.edu.au

School of Mathematics and Statistics UNSW

Sydney, Australia 